\title{
Evaluating the antioxidant potential of new treatments for inflammatory bowel disease using a rat model of colitis
}

A D Millar, D S Rampton, C L Chander, A W D Claxson, S Blades, A Coumbe, J Panetta, C J Morris, D R Blake

\begin{abstract}
Background-Reactive oxygen species may mediate tissue injury in inflammatory bowel disease. Aminosalicylates have antioxidant activity and the antioxidants, superoxide dismutase and allopurinol, are of reported benefit in inflammatory bowel disease.
\end{abstract}

Aim-To develop a convenient technique for testing the antioxidant potential of standard and novel therapeutic agents for use in inflammatory bowel disease.

Methods-Amplified chemiluminescence was used to measure reactive oxygen species production by colonic biopsy specimens from rats with acetic acid induced colitis and to assess the in vitro effect of conventional antioxidants, standard therapies and proposed novel therapies for inflammatory bowel disease. Results-The model was validated by demonstrating that the profile of effects on chemiluminescence of acetic acid induced colitis biopsy specimens given by conventional antioxidants (sodium azide, catalase, copper-zinc superoxide dismutase, dimethyl sulphoxide, $\boldsymbol{N}$-acetylcysteine and ascorbate) and standard therapies (5-aminosalicylate and hydrocortisone) resembled that previously reported using biopsy specimens from ulcerative colitis. Human recombinant manganese superoxide dismutase did not alter chemiluminescence. Two novel compounds, LY231617 (10 $\mathrm{mM})$ and amflutizole $(20 \mathrm{mM})$, reduced chemiluminescence by $98 \%(n=5, p=0.009)$ and $88 \%$ $(n=5, p=0.03)$, respectively.

Conclusions-The similarity of the chemiluminescence responses of colonic biopsy specimens from acetic acid induced colitis and ulcerative colitis to a range of conventional antioxidants and standard treatments suggests that this model is a useful method for testing the antioxidant potential of new therapies for inflammatory bowel disease. The antioxidant actions of dimethyl sulphoxide, ascorbate, and the novel compounds, amflutizole and LY231617 in this model suggest that these agents merit further assessment in the treatment of inflammatory bowel disease.

(Gut 1996; 39: 407-415)

Keywords: ulcerative colitis, antioxidant, acetic acid, aminosalicylates, free radicals, superoxide dismutase.
It is now well recognised that reactive oxygen species (ROS) such as superoxide $\left(\mathrm{O}_{2}{ }^{-}\right)$, the hydroxyl radical $\left(\mathrm{OH}^{\circ}\right)$, hydrogen peroxide $\left(\mathrm{H}_{2} \mathrm{O}_{2}\right)$, hypochlorous acid $(\mathrm{HOCl})$ and oxidant derivatives, such as $N$-chloramines ( $\mathrm{RNHCl}$ ), are produced in excess by the inflamed mucosa in inflammatory bowel disease (IBD) and may be pathogenic. ${ }^{1-4}$ The predominant sources of ROS in the inflamed mucosa are probably activated mucosal phagocytic leucocytes ${ }^{5}$ and episodes of ischaemia reperfusion ${ }^{6}$ (Fig 1). Increased arachidonic acid metabolism ${ }^{7}$ may also lead to excess mucosal ROS production. The resulting oxidant stress may overwhelm the endogenous defences that regulate ROS production during normal metabolism, particularly if there are comparatively low tissue levels of endogenous antioxidants, as in the colonic mucosa. ${ }^{8}$ Consequent tissue injury by $\mathrm{ROS}^{9}$ results from their direct reaction with carbohydrates, lipids, proteins, and DNA, stimulation of phospholipase $\mathrm{A}_{2},{ }^{10}$ 5-lipoxygenase, ${ }^{11}$ and neutrophil chemotaxis, ${ }^{12}$ and activation of transcription factors involved in cytokine release and cell growth, such as $\mathrm{NF}_{\kappa} \mathrm{B},{ }^{13} \mathrm{c}$-fos, c-myc and c-jun. ${ }^{14}$

It has been suggested that the efficacy of current standard treatments is related to their antioxidant actions. ${ }^{4}$ 5-ASA is a potent antioxidant and reduces mucosal ROS production by inflamed human colorectal biopsy tissue in vitro, ${ }^{1}$ as well as mucosal lipid peroxidation in ulcerative colitis (UC) in vivo. ${ }^{15}$ Hydrocortisone and other glucocorticoids do not directly scavenge ROS but their antiinflammatory effects include inhibition of neutrophil function. ${ }^{16}$

Recent, albeit uncontrolled, trials have suggested that specific antioxidant treatment may be therapeutically effective in IBD. In corticosteroid resistant Crohn's disease (CD) a combination of superoxide dismutase and desferrioxamine was reportedly effective, ${ }^{17}$ while allopurinol has been used successfully in acute and chronic pouchitis. ${ }^{18}$ Antioxidants have been used successfully in experimental models of intestinal inflammation. 1920 Compounds with antioxidant activity should therefore be investigated as potential treatments for IBD. There are many compounds with known antioxidant activity and many new potential therapies whose ability to reduce ROS production by inflamed colon is unknown. It would therefore be useful to have a method for screening potential treatments for this action. 


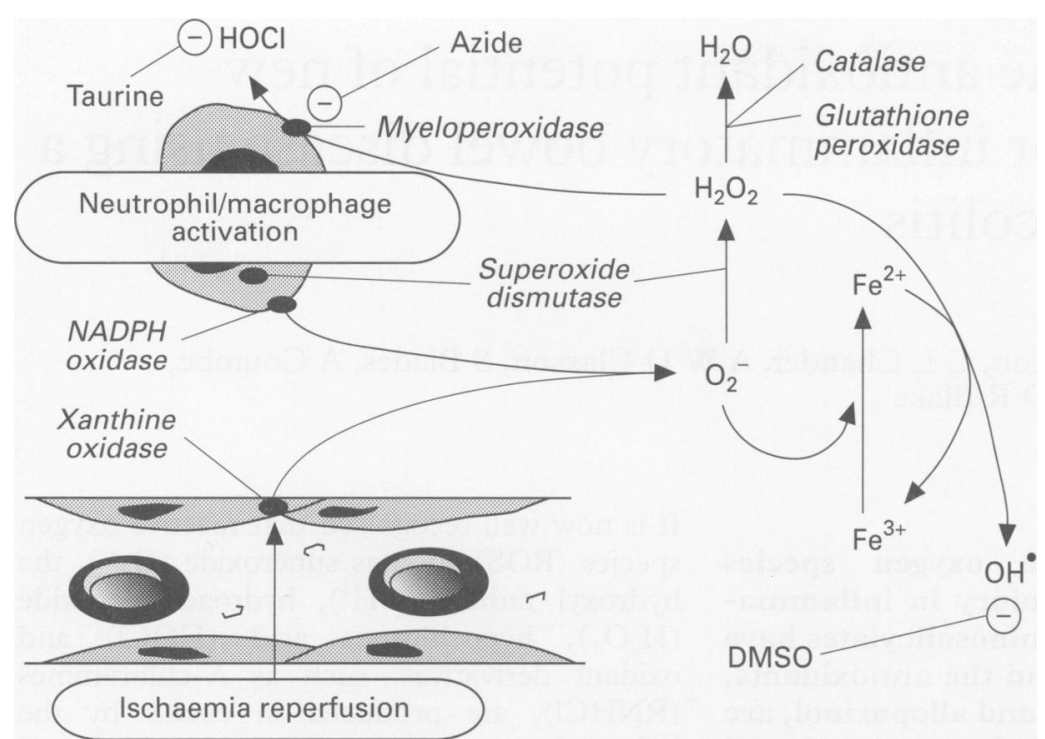

Figure 1: Schematic representation of the major sources of $R O S$ production by the inflamed mucosa in inflammatory bowel disease. Enzymes are shown in italics and three examples of exogenous scavengers in plain text. Superoxide produced by activated neutrophils or ischaemia reperfusion dismutates to hydrogen peroxide, catalysed by superoxide dismutase. Superoxide reduces $\mathrm{Fe}^{3+}$ to $\mathrm{Fe}^{2+}$ thus facilitating the Haber-Weiss reaction by which hydroxyl irons are produced. Hydrogen peroxide is catalysed by myeloperoxidase to hypochlorous acid.<smiles>CCNCc1cc(C(C)(C)C)c(O)c(C(C)(C)C)c1</smiles>

Amflutizole

LY 231617

Figure 2: The chemical structures of amflutizole and LY231617.

Poole, Dorset, unless otherwise stated. The $\mathrm{CuZn}$ superoxide dismutase was from human erythrocytes (specific activity: $3610 \mathrm{U} / \mathrm{mg}$ protein). Catalase was from bovine liver attached to $4 \%$ agarose beads (specific activity: $150000-200000 \mathrm{U} / \mathrm{g}$ agarose). Rh-Mn superoxide dismutase (specific activity: 3500 $\mathrm{U} / \mathrm{mg}$ protein) was provided by Bender, Ges $\mathrm{mbH}$, Vienna, Austria. Amflutizole (3-(3-trifluoromethyl) - 4-aminoisothiazole-5-carboxylic acid) and LY231617 (2,6-bis(1,1-dimethylethyl)-4-[[(1-ethyl)amino]methyl]phenol hydrochloride) (Fig 2) were obtained from Lilly Research Laboratories, Indianapolis, USA.

Table I shows the compounds used and their postulated mechanisms of antioxidant activity.

Luminol (5-amino-2,3-dihydrophthalazine1,4-dione) was maintained as stock solution (50 mg in $1 \mathrm{ml}$ of DMSO) for up to one month. Luminol and lucigenin (N,N'-dimethyl9,9'-biacridinium dinitrate) were prepared prior to the experiment in Dulbecco's phosphate buffered saline (D-PBS) with added calcium $(1.13 \mathrm{mM})$ and glucose $(5 \mathrm{mM})$ and oxygenated for 10 minutes with $95 \% \mathrm{O}_{2}, 5 \%$ $\mathrm{CO}_{2}$ to. maintain tissue viability, following which the $\mathrm{pH}$ was adjusted to $7 \cdot 4$ with $\mathrm{NaOH}$ or $\mathrm{HCl}(1 \mathrm{M})$. oxidants will reduce measurable $\mathrm{ROS}^{23}$. in these studies, however, mucosal scrapings rather than biopsy specimens were used and no comparison was made with human material, thus limiting the comparability with IBD.

This study used full thickness colonic biopsy specimens from acetic acid induced colitis and validated the screening technique by comparing results with previous studies using biopsy specimens from patients with active UC, in which the conventional antioxidants (sodium azide, taurine, dimethyl sulphoxide, $N$-acetylcysteine, ascorbate and the enzymes catalase and $\mathrm{CuZn}$ superoxide dismutase) and two standard therapies for IBD (5-aminosalicylate (5-ASA) and hydrocortisone), were evaluated. ${ }^{12425}$ In addition, we studied the effects of potential new antioxidant approaches with human recombinant manganese superoxide dismutase ( $\mathrm{Rh}-\mathrm{Mn}$ superoxide dismutase) and two novel antioxidants, LY231617 and amflutizole, whose structures are shown in Figure 2.

\section{Methods}

REAGENTS

All chemical reagents were of analytical grade and were obtained from Sigma Chemical,
INDUCTION OF EXPERIMENTAL COLITIS IN RATS Acetic acid induced colitis was induced in male Wistar rats (150-200 g, Charles River, UK) using a modification of the method described by MacPherson and Pfeiffer. ${ }^{26}$ The animals were fasted for 16 hours with access to water ad libitum. Each rat was sedated by brief respiration of $20-25 \% \quad \mathrm{CO}_{2}$ followed by anaesthesia with $35 \mathrm{mg} / \mathrm{kg}$ intraperitoneal pentobarbital. An infant feeding tube (Pennine Healthcare FT-1608/40, outside diameter 2 $\mathrm{mm}$ ) was inserted into the colon to $8 \mathrm{~cm}$ and $2 \mathrm{ml}$ of acetic acid (3\% v/v in $0.9 \%$ saline) or saline alone (control animals) infused into the colon. The acetic acid/saline was retained in the colon for 30 seconds, after which fluid was withdrawn. The rats were killed at 24 hours by $\mathrm{CO}_{2}$ asphyxiation.

\section{ASSESSMENT OF COLITIS}

\section{Macroscopic scoring}

At postmortem laparotomy, $6 \mathrm{~cm}$ of colon extending proximally from $2 \mathrm{~cm}$ above the anal margin was removed, split longitudinally, pinned out on card, and the macroscopic 
TABLE I Study compounds

\begin{tabular}{|c|c|c|c|c|}
\hline $\begin{array}{l}\text { Drug/compound } \\
\text { (study concentration) }\end{array}$ & Antioxidant action (reference) & $\begin{array}{l}\text { Soluble in } \\
\text { aqueous } \\
\text { solution }\end{array}$ & $\begin{array}{l}\text { Chemiluminescence } \\
\text { amplifier }\end{array}$ & $\begin{array}{l}\text { Control } \\
\text { solution }\end{array}$ \\
\hline \multicolumn{5}{|l|}{ Conventional antioxidants } \\
\hline Catalase $(3000 \mathrm{U} / \mathrm{ml})$ & $\Downarrow \mathrm{H}_{2} \mathrm{O}_{2}$ & + & Luminol & $\mathrm{HI}^{\star}$ enzyme \\
\hline Taurine $(20 \mathrm{mM})$ & $\Downarrow \mathrm{HOCl}^{56}, \mathrm{OH}^{\cdot 57}$ & + & Luminol & D-PBS \\
\hline $\mathrm{CuZn}$ superoxide dismutase $(30 / 300 \mathrm{U} / \mathrm{ml})$ & $\Downarrow \mathrm{O}_{2}^{--}$ & + & Lucigenin & $\mathrm{HI}^{\star}$ enzyme \\
\hline Dimethylsulphoxide $(1 / 5 / 10 \%)$ & OH' 58 & + & Luminol & D-PBS \\
\hline$N$-Acetylcysteine $(20 \mathrm{mM})$ & $\Uparrow$ Glutathione peroxidase $/ \Downarrow \mathrm{HOCl}^{46}$ & + & Luminol & D-PBS \\
\hline Ascorbate $(20 \mathrm{mM})$ & $\Downarrow \mathrm{HOCl}^{47} /$ thiyl/peroxyl/O ${ }_{2}^{\cdot-46}$ & + & Luminol & D-PBS \\
\hline \multicolumn{5}{|l|}{ Standard treatments in IBD } \\
\hline 5-ASA $(1 / 10 / 20 \mathrm{mM})$ & $\Downarrow \mathrm{OH}^{\cdot 59} / \mathrm{O}_{2}{ }^{-6}{ }^{60} / \mathrm{HOCl}^{61} / \mathrm{Fe}$ chelator ${ }^{62}$ & + & Luminol & D-PBS \\
\hline Hydrocortisone (1 mM) & $\Downarrow$ Neutrophil function 16 & + & Luminol & D-PBS \\
\hline \multicolumn{5}{|l|}{ Proposed treatments in IBD } \\
\hline $\mathrm{Rh}-\mathrm{Mn}$ superoxide dismutase $(300 \mathrm{U} / \mathrm{ml})$ & $\Downarrow \mathrm{O}_{2} \cdot-$ & + & Lucigenin & $\mathrm{HI}^{\star}$ enzyme \\
\hline Amflutizole $(1 / 10 / 20 \mathrm{mM})$ & Inhibits xanthine oxidase 52 & - & Luminol & D-PBS \\
\hline LY231617 (1/10 mM) & Not known & - & Luminol & D-PBS \\
\hline
\end{tabular}

$\star=$ Heat inactivated, $\Downarrow=$ scavenges or inhibits, $\Uparrow=$ stimulates. All compounds and controls were dissolved in Dulbecco's phosphate buffered saline (D-PBS). For most compounds control was D-PBS alone.

appearances of the colonic mucosa scored on a scale adapted from Morris et $a l^{27}$ ranging from 0-4: (0) no macroscopic change, (1) mucosal erythema alone, (2) mild mucosal oedema, slight bleeding or small erosions, (3) moderate oedema, bleeding ulcers or erosions, (4) severe ulceration/erosions, oedema, and tissue necrosis. Intermediate values reflected intermediate appearances.

\section{Microscopic scoring}

In some experiments randomly distributed full thickness biopsy specimens were fixed in $10 \%$ formol saline prior to wax embedding, sectioning, and staining with haematoxylin and eosin. Biopsy specimens $(n=27)$ from 14 animals were scored by a histopathologist blinded to the macroscopic score and chemiluminescence responses of the sections, using the scoring system shown in Table II.

CHEMILUMINESCENCE ASSAY

Luminol and lucigenin react with oxidants, such as ROS, to form 3-aminophthalate and $\mathrm{N}$-methylacridone, respectively. Electrons in the luminol and lucigenin are raised to higher energy levels during the reaction with oxidants. On reverting to the ground state, energy is

TABLE II Histological assessment of full thickness biopsy specimens from acetic acid colitis in rats

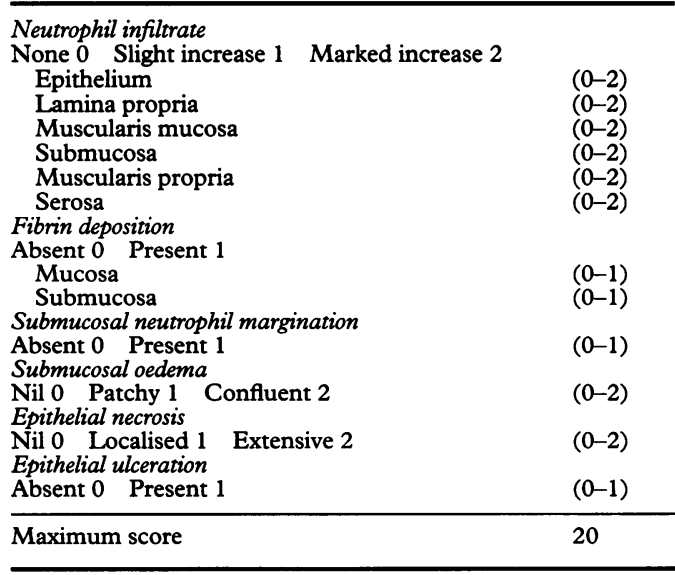

released in the form of photons, which are detected by the photomultiplier tubes and photon detectors that comprise the scintillation counter. ${ }^{28}$ Lucigenin responds more specifically to superoxide than luminol ${ }^{29}$ and was therefore used to detect changes in chemiluminescence induced by superoxide dismutase. Luminol and lucigenin were used at the same concentration $(300 \mu \mathrm{M})$ as used in previous studies using human biopsy specimens. ${ }^{1}$

Full thickness biopsy specimens were taken at random by cutting cross sectional strips (median (IQR) wet weight; $44(31-78) \mathrm{mg}$ ) of the dissected, inflamed colon. Biopsy specimens from colons of macroscopic score $\geqslant 2$ were used for drug studies. Such samples were initially placed in pre-oxygenated $(95 \%$ $\mathrm{O}_{2}, 5 \% \mathrm{CO}_{2}$ for 10 minutes) D-PBS, with added calcium ( $1.13 \mathrm{mM})$ and glucose ( $5 \mathrm{mM})$ at ambient temperature and then transferred to $300 \mu \mathrm{M}$ luminol or lucigenin immediately prior to assessment of the chemiluminescence response, which was measured in a Packard Tri Carb 1600 CA liquid scintillation counter analyser operated in 'the out-of-coincidence' mode for two minutes.

ASSESSMENT OF EFFECTS OF TEST COMPOUNDS ON CHEMILUMINESCENCE RESPONSE

All compounds and appropriate controls were prepared prior to the experiment and adjusted to $\mathrm{pH} 7 \cdot 4$ by addition of $1 \mathrm{M} \mathrm{NaOH}$ or $\mathrm{HCl}$. Controls for these experiments were vehicle (D-PBS) for all compounds except $\mathrm{CuZn}$ superoxide dismutase, $\mathrm{Rh}-\mathrm{Mn}$ superoxide dismutase, and catalase, in which heat inactivated enzyme was used (Table I). Heat inactivated enzymes were prepared by heating in D-PBS at $100^{\circ} \mathrm{C}$ for two hours. ${ }^{30}$

Water soluble compounds

The effect of test compounds on the chemiluminescence response of rat acetic acid induced colitis biopsy specimens was compared with the response of a paired biopsy to the control for that compound. The chemiluminescence response was measured in $1.8 \mathrm{ml}$ 
of $300 \mu \mathrm{M}$ luminol or lucigenin and then immediately repeated after addition of $200 \mu \mathrm{l}$ of the test compound, at 10 times the final concentration, or appropriate control. After counting, samples were blotted, weighed, and in some experiments, placed in $10 \%$ formol saline for subsequent histological examination.

Water insoluble compounds

LY231617 and amflutizole were insoluble in aqueous solution at the required concentrations. Suspensions were therefore prepared in $10 \%$ acacia dissolved in D-PBS, oxygenated for 10 minutes in $95 \% \mathrm{O}_{2}, 5 \% \mathrm{CO}_{2}$ and adjusted to $\mathrm{pH} 7 \cdot 4$ with $1 \mathrm{M} \mathrm{NaOH}$. Acacia is a demulcent gum used to suspend or emulsify water insoluble pharmacological agents. Oxygenation of these suspensions was required as the biopsy specimens were transferred to the acacia suspensions between the chemiluminescence counts rather than remaining in the vial as with the water soluble agents. After the initial chemiluminescence count, as described above, five specimens were placed together in $10 \%$ acacia with or without the addition of the test compound in suspension. The specimens were grouped to avoid disparity in dosage exposure due to heterogeneity in the suspension. Incubation was carried out for 30 minutes in a shaking water bath at $37^{\circ} \mathrm{C}$. At the end of this time the samples were removed, washed twice in oxygenated D-PBS to remove excess acacia, and luminol amplified chemiluminescence recounted. As a comparator, 5-ASA was also assessed in acacia.

\section{CALCULATIONS AND STATISTICS}

Chemiluminescence response of biopsy specimens Luminol and lucigenin amplified chemiluminescence is expressed as the number of photons $/ \mathrm{min} / \mathrm{mg}$ wet weight of tissue after subtraction of the background count. The background count was taken as the average photons/min for two vials of each 12 analysed containing $300 \mu \mathrm{M}$ luminol or lucigenin in oxygenated D-PBS alone. Comparisons between groups of untreated control animals, saline treated animals, and acetic acid induced colitis were made using the Kruskal-Wallis and Dunn's multiple comparison tests.

To assess the variability of the chemiluminescence response of colonic biopsy specimens from acetic acid induced colitis, the coefficient of variation was calculated from the chemiluminescence response in luminol produced by two to six biopsy specimens from each of 22 rats. $^{31}$ Comparisons between animals are expressed as the median and interquartile range (IQR) of the coefficient of variation. A similar calculation for the response to lucigenin was made in two to six specimens from each of 10 animals.

The macroscopic appearances, the histological score, and the chemiluminescence response were compared using Spearman's rank correlation coefficient.

\section{Effect of water soluble compounds}

For each compound at each concentration a pair of biopsy specimens from each of $\geqslant 5$ rats was used to assess the percentage change in chemiluminescence. The chemiluminescence counts before and after addition of test compound are given as $t_{1}$ and $t_{2}$, respectively, and for the specimen treated with control, $c_{1}$ and $c_{2}$, respectively. To take account of small changes in control values during the assay, the change in chemiluminescence produced by the test compound on one biopsy specimen was adjusted by the change in chemiluminescence produced by the control solution with its pair. Accordingly, the percentage change in chemiluminescence response induced by the test compound $(\Delta \% \mathrm{t})$ is calculated as follows: $\Delta \% \mathrm{t}=\left(\left(\mathrm{t}_{2} \times \mathrm{c}_{1} / \mathrm{c}_{2}\right)-\mathrm{t}_{1}\right) \times 100$.

The results are expressed as median \% (IQR). The difference in chemiluminescence response between biopsy specimens treated with test compounds and controls is calculated by comparing the percentage change in chemiluminescence induced by the test agent and control using the Wilcoxon signed rank test for paired variables. A $p$ value of less than 0.05 (two tailed) was taken as significant.

To investigate the variability of the change in chemiluminescence response produced by vehicle alone, the coefficient of variation of the response to D-PBS (control for most of the compounds tested) was calculated for two to six biopsies from each of 10 animals and the median (IQR) expressed for the coefficient of variation of the luminol amplified chemiluminescence response to D-PBS in all $10 .{ }^{31}$

\section{Effect of water insoluble compounds}

Results are expressed as the mean percentage change in chemiluminescence for each group of biopsy specimens exposed to the test compound after correction of the initial count for the percentage change induced by acacia alone (control). No range is given because grouping the biopsy specimens together resulted in loss of animal identity. The chemiluminescence counts before and after exposure to the test compound were compared using the Mann-Whitney $U$ test. A $p$ value of less than 0.05 (two tailed) was taken as significant.

To investigate the variability of the chemiluminescence response induced by incubation of biopsy specimens in $10 \%$ acacia, the median coefficient of variation was calculated for eight groups of five biopsies from five rats incubated in $10 \%$ acacia alone that were used as controls in experiments assessing the effect of water insoluble compounds.

\section{Results}

VALIDATION OF THE ACETIC ACID INDUCED COLITIS MODEL

Chemiluminescence response in acetic acid induced colitis compared with controls

We compared the chemiluminescence response of 71 colonic biopsy specimens from 23 rats 
with acetic acid induced colitis to eight noninflamed colonic biopsy specimens from four rats given intracolonic $0.9 \%$ saline and 22 biopsy specimens from 10 untreated control rats (Fig 3). The median (IQR) for each of these groups was 17449 (6498-38 880) photons $/ \mathrm{min} / \mathrm{mg}, 74(0-154)$, and $0(0-66)$ respectively. For lucigenin amplified chemiluminescence, 26 colonic biopsy specimens from 10 rats with acetic acid induced colitis were compared with eight specimens from four rats given $0.9 \%$ saline and to 10 specimens from seven untreated controls (Fig 3). The median (IQR) for these groups was 819 (518-1471) photons/min/mg, 347 (157-759), and 143 (48-227), respectively. Biopsy specimens from acetic acid induced colitis produced significantly more luminol amplified chemiluminescence than specimens from saline treated and untreated controls and more lucigenin amplified chemiluminescence than untreated controls $(p<0.001)$ (Fig 3). There was no difference in the chemiluminescence response of colonic tissue from the two control groups.

\section{Chemiluminescence related to the severity of inflammation}

To assess the relation between ROS production as detected by chemiluminescence and inflammation in the acetic acid induced colitis model we compared the luminol amplified chemiluminescence response of full thickness biopsy specimens with the macroscopic colonic appearances and the histological grade of inflammation in rats with acetic acid induced colitis. Figures 4 and 5 show significant positive correlations between the macroscopic and histological scores, respectively, of

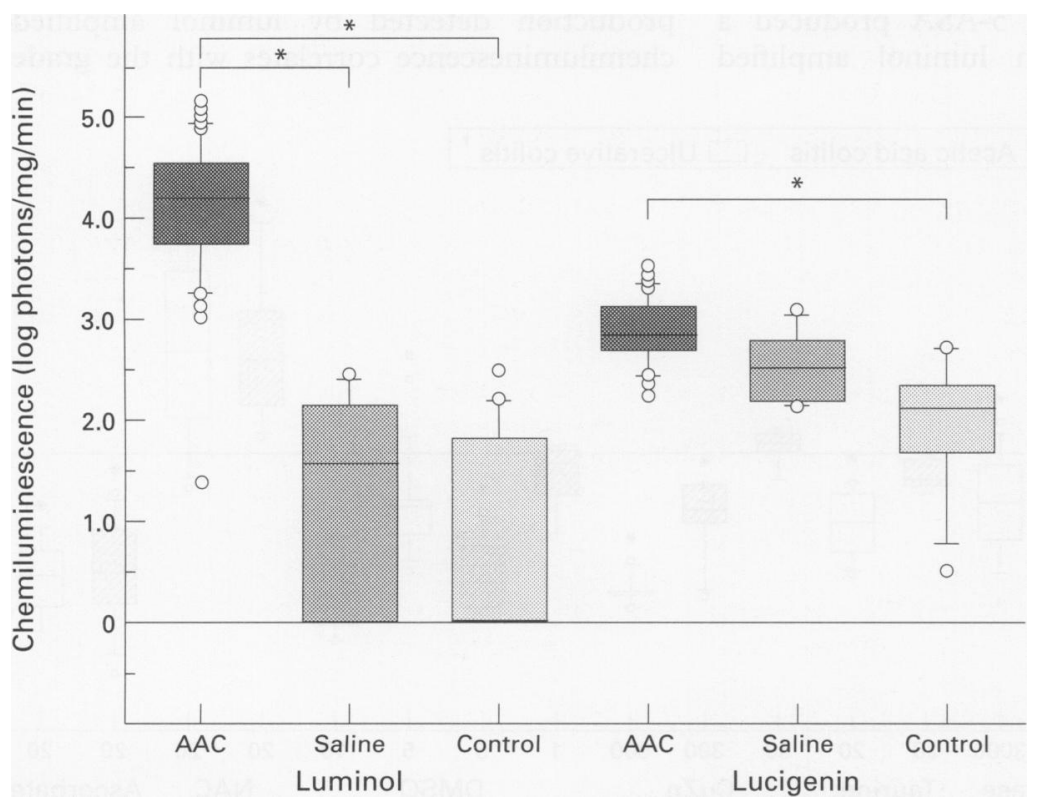

Figure 3: Log transformed luminol and lucigenin amplified chemiluminescence counts in full thickness colonic biopsy specimens from rats pre-treated with 3\% intracolonic acetic acid (AAC) or $0.9 \%$ saline (Saline), assessed at 24 hours and in untreated control animals (Control). For luminol 71 colonic biopsy specimens from 23 rats with acetic acid induced colitis were compared with eight colonic biopsy specimens from four rats given intracolonic $0.9 \%$ saline and 22 biopsy specimens from 10 untreated controls. For lucigenin amplified chemiluminescence, 26 specimens from 10 animals with acetic acid induced colitis were compared with eight specimens from four animals given $0.9 \%$ saline and 10 specimens from seven untreated controls. ${ }^{\star} p<0 \cdot 001$, Dunn's multiple comparison test.

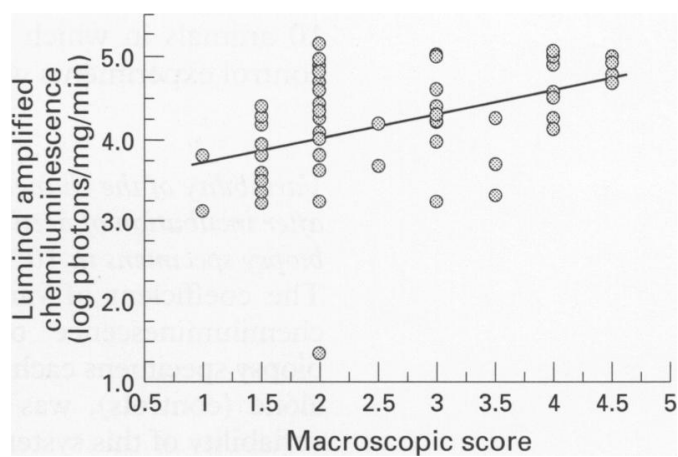

Figure 4: Correlation of luminol amplified

chemiluminescence with the macroscopic score in 71 biopsy specimens from 23 rats pre-treated with $3 \%$ acetic acid induced colitis at 24 hours. Data shown as macroscopic score against the log of the chemiluminescence, showing the regression line, $y=0 \cdot 3 x+3 \cdot 4, r=+0 \cdot 5, p=0 \cdot 0001$, Spearman's rank correlation coefficient.

inflamed rat colons and the chemiluminescence response of tissue from the same specimens. The macroscopic and histological score were also positively correlated $(r=+0 \cdot 7$, $\mathrm{p}=0 \cdot 0001$; data not shown).

\section{Variability of the initial chemiluminescence} response

The median (IQR) of the coefficient of variation of the initial chemiluminescence response of two to six specimens from 22 rats, with acetic acid induced colitis, to luminol was $66(39-83) \%$ and of two to six specimens from 10 rats to lucigenin was $29(17-49) \%$. These variations are small in comparison to the percentage increase in luminol and lucigenin amplified chemiluminescence response of acetic acid induced colitis biopsy specimens compared with control (mean, $+20000 \%$ and $+500 \%$, respectively).

Variability of the change in chemiluminescence after addition of Dulbecco's PBS

The coefficient of variation of the change in luminol amplified chemiluminescence resulting from incubation in D-PBS (control for most of the experiments) calculated from the

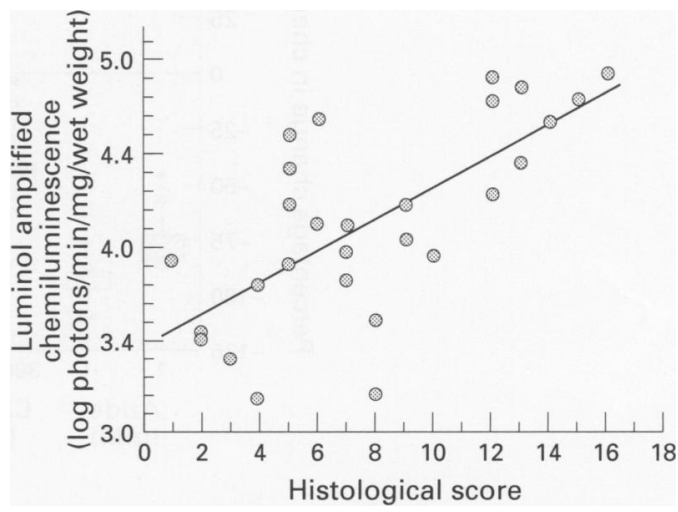

Figure 5: Correlation of luminol amplified

chemiluminescence with the histological score (see Table II) in 27 biopsy specimens from 14 rats pre-treated with $3 \%$ acetic acid induced colitis at 24 hours showing the regression line, $y=0 \cdot 1 x+3 \cdot 5, \mathrm{r}=+0 \cdot 7, p=0 \cdot 0004$, Spearman's rank correlation coefficient. 
10 animals in which there were two or more control experiments was $18(11-41) \%$.

Variability of the change in chemiluminescence after incubation of acetic acid induced colitis biopsy specimens in $10 \%$ acacia

The coefficient of variation for the change in chemiluminescence of eight groups of five biopsy specimens each, incubated in $10 \%$ acacia alone (controls), was $48 \%$, which reflects the variability of this system compared with incubation in D-PBS.

COMPARISON OF RESPONSE OF BIOPSY SPECIMENS FROM ACETIC ACID INDUCED COLITIS AND UC TO CONVENTIONAL ANTIOXIDANTS

Significant reductions in luminol amplified chemiluminescence in the acetic acid induced colitis model were observed with sodium azide $(-83(-85$ to -73$) \% \mathrm{p}=0.03)$, catalase $(-43$ $(-47$ to -41$) \%, p=0.03), 10 \%(1.28 \mathrm{M})$ DMSO ( $-67(-78$ to $-50 \%, \mathrm{p}=0.03)$, and ascorbate $(-54(-69$ to -35$) \%, p=0.01)$ and in lucigenin amplified chemiluminescence with $\mathrm{CuZn}$ superoxide dismutase $(-26 \quad(-32$ to $-15) \%, p=0.03$ ) (Fig 6). $N$-acetylcysteine produced no significant change in luminol amplified chemiluminescence. The profile of responses in acetic acid induced colitis demonstrate a striking resemblance to that previously seen in UC. ${ }^{124}{ }^{25}$ With one compound, taurine, a small, but significant, reduction was produced in UC biopsy specimens ${ }^{1}$ but not in acetic acid induced colitis.

COMPARISON OF RESPONSE OF BIOPSY SPECIMENS FROM ACETIC ACID INDUCED COLITIS AND UC TO STANDARD TREATMENTS As for UC specimens, 5-ASA produced a significant reduction in luminol amplified chemiluminescence response in acetic acid induced colitis biopsy specimens (maximum $-88(-89$ to -70$) \%$ at $20 \mathrm{mM} 5-\mathrm{ASA}, \mathrm{p}=0.03)$ with a limited dose response producing an estimated $\mathrm{IC}_{50}$ of $4 \mathrm{mM}$ (Fig 7). Hydrocortisone did not alter luminol amplified chemiluminescence in either acetic acid induced colitis or UC biopsy specimens ${ }^{24}$ (Fig 7).

RESPONSE OF BIOPSY SPECIMENS FROM ACETIC ACID INDUCED COLITIS TO POTENTIAL NEW TREATMENTS FOR IBD

Rh-Mn superoxide dismutase had no demonstrable antioxidant activity in this system, altering the lucigenin amplified and luminol amplified chemiluminescence response, respectively by $-9(-17$ to +3$) \%$ and $+17(+10$ to +37$) \%$, respectively. However, two novel water insoluble antioxidant compounds, LY231617 (10 mM) and amflutizole (20 mM), produced noticeable reductions in luminol amplified chemiluminescence after incubation of the specimens in suspensions of the compounds in $10 \%$ acacia (means, $-98 \%$, $\mathrm{p}=0.009$ and $-88 \%, \mathrm{p}=0.028$, respectively) (Fig 8). 5-ASA (20 mM), which had previously been shown to reduce luminol amplified chemiluminescence in aqueous solution, reduced the chemiluminescence by a similar amount (mean, $83 \% \mathrm{p}=0.002$ ) when tested in acacia.

\section{Discussion}

These findings show that full thickness colonic biopsy specimens from rats with acetic acid induced colitis produce increased levels of ROS, as detected by amplified chemiluminescence, compared with control tissue. In addition, we have found that the level of ROS production detected by luminol amplified chemiluminescence correlates with the grade

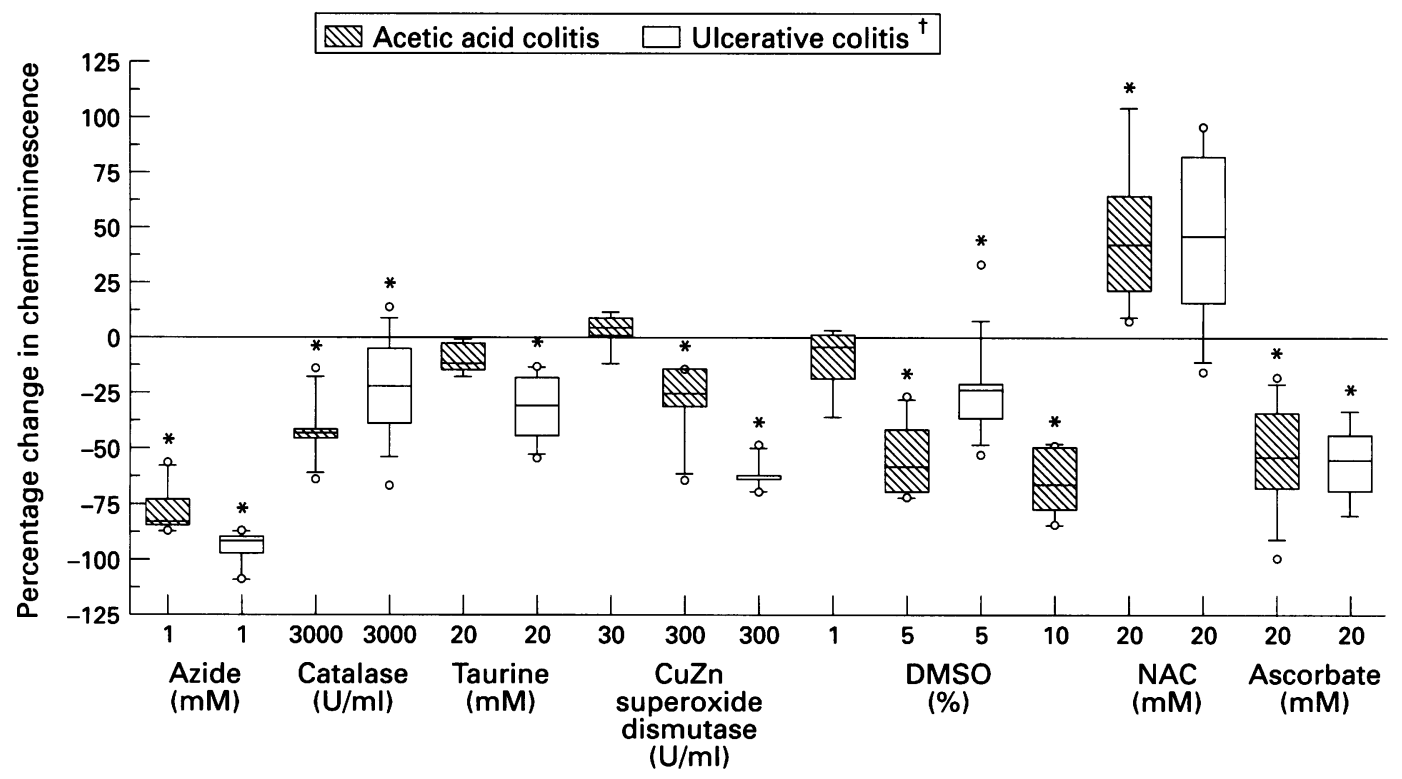

Figure 6: The percentage change in chemiluminescence response of inflamed biopsy specimens from acetic acid induced colitis to conventional antioxidants compared with results using mucosal biopsy specimens from active UC ( $\dagger$ data redrawn from references ${ }^{124}$ 25). Chemiluminescence was measured before and after exposure to test compound and compared with a second biopsy specimen exposed to vehicle ( $n \geqslant 5 ;{ }^{\star}$ denotes $p<0.05$ drug compared with vehicle, Wilcoxon signed rank test).

Luminol was used as the amplifier for chemiluminescence for all compounds except CuZn superoxide dismutase, for which lucigenin was used. $N A C=\mathrm{N}$-acetylcysteine. 


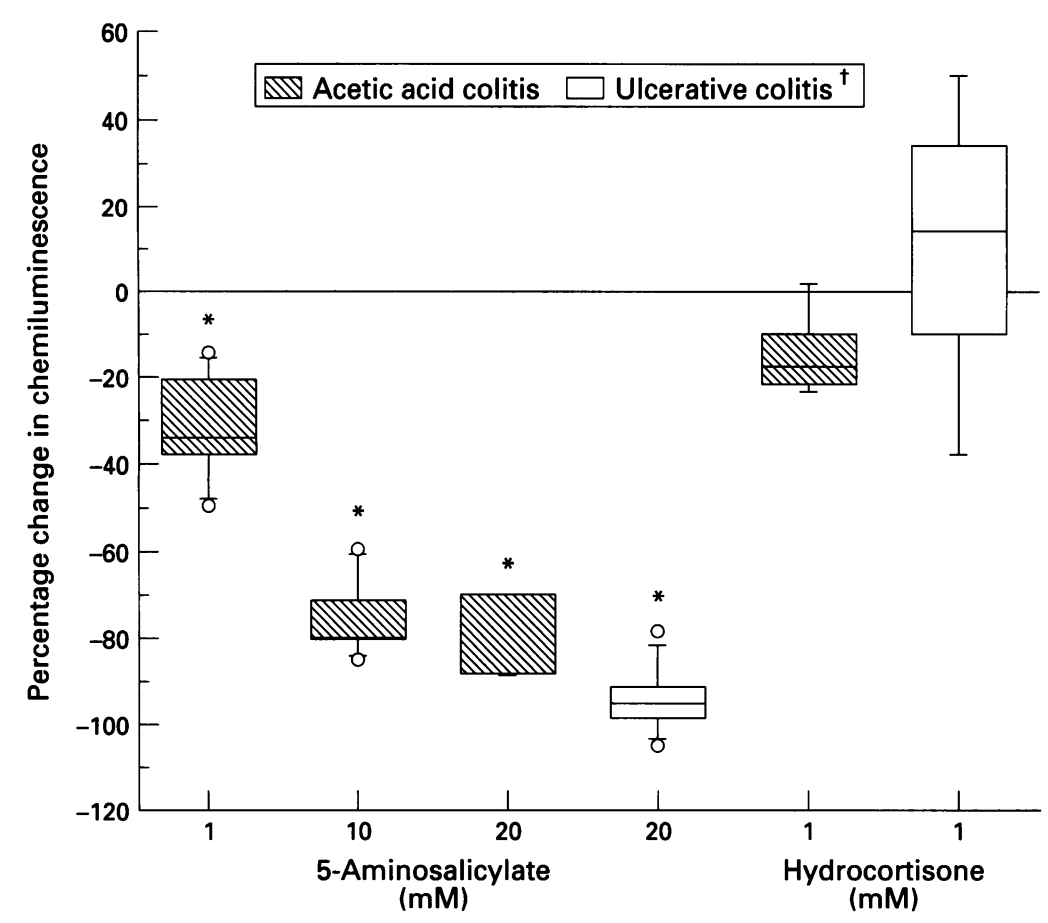

Figure 7: The percentage change in the luminol amplified chemiluminescence response of inflamed biopsy specimens from acetic acid induced colitis to standard treatments in IBD compared with results using mucosal biopsies from active UC (†data redrawn from references ${ }^{12425}$ ). Chemiluminescence was measured before and after exposure to test compound and compared with a second biopsy specimen exposed to vehicle $\left(n \geqslant 5 ;{ }^{\star}\right.$ denotes $p<0.05$ drug compared with vehicle, Wilcoxon signed rank test).

of mucosal inflammation, as assessed by the macroscopic and histological score. These findings confirm previous studies using mucosal scrapings from rat acetic acid induced colitis. $^{22}$

We have now shown that colonic biopsy specimens from the acetic acid induced colitis model respond to conventional antioxidants and standard treatments for IBD, 5-ASA, and hydrocortisone, in a similar fashion to mucosal specimens from patients with active UC. ${ }^{12425}$ The estimated $\mathrm{IC}_{50}$ of 5-ASA in this model compares favourably with the intraluminal concentrations recorded after oral administration of aminosalicylates (5-10 $\mathrm{mM})$ in UC. $^{32}$ Hydrocortisone had no antioxidant
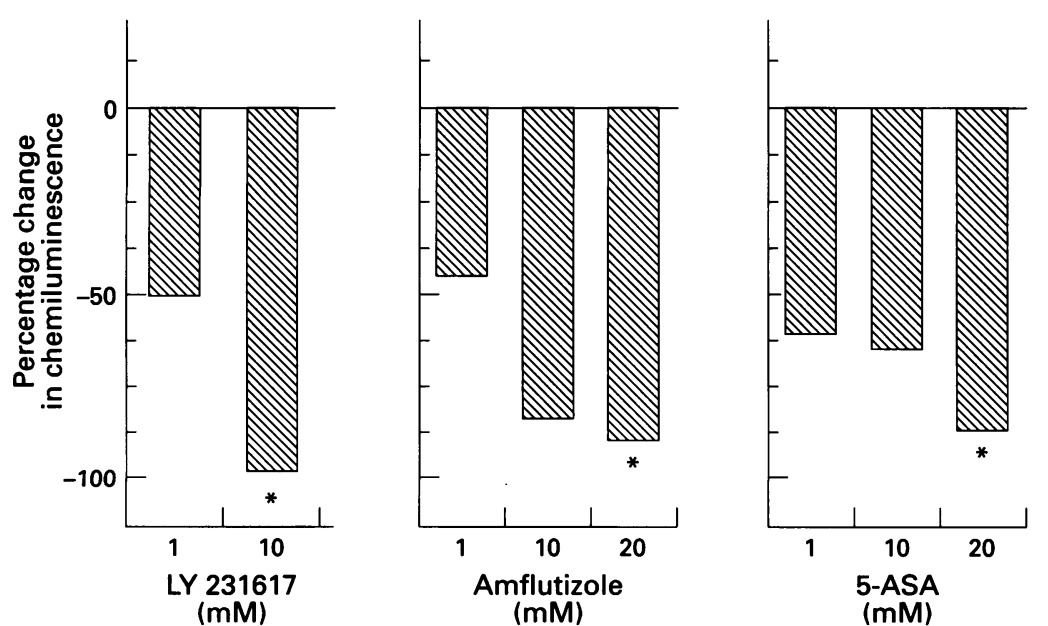

Figure 8: Mean percentage change in luminol amplified chemiluminescence response of inflamed biopsy specimens from acetic acid induced colitis to proposed new treatments, LY231617, amflutizole, and to 5-ASA. Chemiluminescence was measured before and after incubation of colonic biopsy specimens in a suspension of the test compound in $10 \%$ acacia for 30 minutes and compared with specimens exposed to acacia alone ( $n=5 ; \star$ denotes $p<0.05$ drug compared with vehicle, Mann-Whitney $U$ test). activity in the present short-term in vitro experiments in this model or with UC biopsy specimens. ${ }^{24}$ However, other studies, using prolonged incubation with dexamethasone in vitro, have shown inhibition of superoxide generation by stimulated phagocytes ${ }^{33}$ : the therapeutic effect of hydrocortisone in vivo may thus be partly due to an indirect antioxidant action by inhibition of neutrophil function. ${ }^{16}$

Of the conventional antioxidants examined, the greatest inhibition occurred with azide, a potent inhibitor of myeloperoxidase, ${ }^{28}$ which also inhibits other haem proteins such as catalase and quenches singlet oxygen and the hydroxyl ion. ${ }^{34}$ Although the antioxidant action of azide could result from a cytotoxic effect, azide has been shown to reduce the luminol amplified chemiluminescence of stimulated neutrophils to a greater effect when added before the stimulus $(100 \%)$ than after $(20 \%),{ }^{35}$ an effect that would not occur if the reduction in chemiluminescence was purely due to cytotoxicity.

Superoxide is produced by the activated neutrophil, partly as the result of NADPH activity, ${ }^{36}$ and as a result of episodes of ischaemia reperfusion, which may be important in the pathogenesis of $\mathrm{IBD}^{37}$ (Fig 1). Superoxide generation has been located to the epithelium, vascular endothelium, and infiltrating mononuclear cells in inflamed UC mucosa $^{38}$ and levels of superoxide dismutase are reduced in inflamed intestinal resection tissue from patients with IBD. ${ }^{39}$ Administration of exogenous superoxide dismutase is of benefit in vivo in the acetic acid induced colitis model ${ }^{19}$ and there is preliminary, uncontrolled, clinical evidence that liposomal encapsulated $\mathrm{CuZn}$ superoxide dismutase may be of benefit in $\mathrm{CD}^{17}$ and UC. ${ }^{40}$ The present studies using acetic acid induced colitis biopsy specimens support previous findings showing that $\mathrm{CuZn}$ superoxide dismutase reduces lucigenin amplified chemiluminescence by mucosal biopsy specimens in vitro from UC. ${ }^{24}$

$\mathrm{Rh}-\mathrm{Mn}$ superoxide dismutase has theoretical advantages over $\mathrm{CuZn}$ superoxide dismutase as a potential therapeutic agent for IBD because of its longer half life $\mathrm{e}^{41}$ and its human origin should lessen the risk of in vivo antigenicity. In two models of chronic inflammation, adjuvant induced arthritis and bleomycin induced lung damage, Rh-Mn superoxide dismutase was shown to be more effective than $\mathrm{CuZn}$ superoxide dismutase. ${ }^{42}$ Mn superoxide dismutase has been shown to reduce luecocyte adhesion in a model of intestinal ischaemia ${ }^{43}$ and, in high dose, to improve trinitrobenzene sulphonic acid/ ethanol induced colitis. ${ }^{20}$ The failure of Rh-Mn superoxide dismutase to reduce ROS and production by acetic acid induced colitis biopsy specimens in vitro may be due to poor tissue penetration of this large tetradimer: this may also limit its potential use in vivo.

Hydrogen peroxide, produced by superoxide dismutation and peroxidases, is injurious to the rectal mucosa in humans when applied topically ${ }^{44}$ and intraperitoneal catalase improves inflammation in rat acetic acid induced 
colitis, ${ }^{19}$ suggesting that hydrogen peroxide and its metabolites may contribute to colonic inflammation. An antioxidant effect for catalase can be demonstrated in both acetic acid induced colitis and UC biopsy specimens, ${ }^{1}$ further supporting the suggestion that ROS produced by neutrophils are important in IBD.

$\mathrm{N}$-acetylcysteine replenishes intracellular stores of glutathione, is a weak scavenger of superoxide and hydroperoxide but reacts avidly with hypochlorous acid and the hydroxyl radical. ${ }^{45}$ However, $\mathrm{N}$-acetylcysteine was ineffective in reducing ROS production by acetic acid induced colitis or UC biopsy specimens under the present experimental conditions. ${ }^{24}$

The physiological level of ROS is regulated by both enzymatic systems and direct oxygen scavengers, such as ascorbate, which reacts rapidly with superoxide, the hydroxyl radical, ${ }^{46}$ and hypochlorous acid. ${ }^{47}$ Ascorbate levels are decreased in the plasma in IBD. ${ }^{48} 49$ In this investigation ascorbate produced similar reductions in chemiluminescence in acetic acid induced colitis and human UC biopsy specimens. ${ }^{24}$ However, ascorbate is readily oxidised to the potentially damaging ascorbate radical in the presence of iron, ${ }^{50}$ present in the bowel lumen in millimolar quantities. ${ }^{3}$ This reaction could limit the potential usefulness of ascorbate as a treatment in UC.

The predominant antioxidant action of DMSO in vitro is scavenging of the hydroxyl ion. ${ }^{5}$ As the hydroxyl ion is extremely reactive, the local concentration of any scavenger would probably have to be prohibitively high to compete with biological molecules in the vicinity of its production. This may explain evidence from in vivo studies in which oral $5 \%$ $(0.64 \mathrm{M})$ DMSO failed to improve acetic acid induced colitis. ${ }^{22}$ Nevertheless, the antioxidant effects of DMSO obtained here, and in UC biopsy specimens, ${ }^{1}$ suggest that evaluation of its potential efficacy when given in enema formulation in high concentration to patients with distal UC may be worthwhile.

The hypochlorite scavenger taurine had little effect on ROS production by UC biopsy specimens $^{1}$ and no effect in this model, possibly because the reaction product of hypochlorous acid and taurine, taurine- $N$ chloramine, is itself an oxidant. ${ }^{29}$

The novel agent, LY231617, protects against cerebral ischaemia in an animal model in vivo and ameliorates hydrogen peroxide induced neuronal damage in vitro. ${ }^{51}$ The potent antioxidant activity shown here suggests that it should be further evaluated as treatment in UC, perhaps initially in an enema or suppository formulation to achieve the necessary intraluminal concentration (10 $\mathrm{mM})$.

We studied another antioxidant, amflutizole, which is a non-competitive inhibitor of xanthine oxidase and has also shown efficacy in a model of neuronal ischaemia. ${ }^{52}$ Ischaemia reperfusion with increased superoxide generation through xanthine oxidase activation ${ }^{36}$ may have a pathogenic role in IBD. Multifocal vascular microinfarction is a feature of $\mathrm{CD}^{37}$ and microvascular thrombi have been detected in both $\mathrm{UC}$ and $\mathrm{CD} .{ }^{53}$ The competitive xanthine oxidase inhibitor, allopurinol, has been of reported benefit in pouchitis. ${ }^{18}$ In the present studies amflutizole was at least as effective as 5-ASA in reducing the chemiluminescence response of biopsy specimens from acetic acid induced colitis. Xanthine oxidase inhibition with amflutizole should, therefore, be further explored as a therapeutic option in IBD; again initial studies using topical treatment in distal UC would be desirable.

In conclusion, this in vitro system using biopsy specimens taken from rats with acetic acid colitis provides a convenient method for the screening of the antioxidant potential of new treatments for IBD. Agents that reduce ROS production by inflamed human colorectal biopsy specimens, such as $\mathrm{CuZn}$ superoxide dismutase, catalase, and DMSO also ameliorate inflammation in acetic acid induced colitis. Previous workers have suggested that the ROS production in both $\mathrm{UC}^{1}$ and acetic acid induced colitis ${ }^{19} 22$ is largely mediated by neutrophils, and the results here with azide, catalase, and $\mathrm{CuZn}$ superoxide dismutase support this proposal. The additional evidence of an antioxidant effect with the xanthine oxidase inhibitor, amflutizole, and previous evidence that allopurinol reduces ROS production by inflamed colonic biopsy specimens from acetic acid induced colitis ${ }^{22}$ suggests that, at least in this animal model, ischaemia reperfusion may also contribute to mucosal oxidant stress.

The limited antioxidant effect of antioxidant enzymes in both acetic acid induced colitis and UC biopsy specimens ${ }^{1}$ may be because of poor tissue penetration or inactivation by hypochlorous acid $^{54}$ produced by activated neutrophils; these factors may restrict their therapeutic potential.

Of the agents assessed in this study, dimethyl sulphoxide, ascorbate, amflutizole, and LY231617 seem most suitable for further evaluation in IBD.

This work was supported by grants from Eli-Lilly and Co, USA and Bender and $\mathrm{Co}$, Ges mbH, Vienna, Austria.

1 Simmonds NJ, Allen RE, Stevens TRJ, van Someren RNM, Blake DR, Rampton DS. Chemiluminescence assay of mucosal reactive metabolities in inflammatory bowel disease. Gastroenterology 1992; 103: 186-96.

2 Sedghi S, Fields JZ, Klamut M, et al. Increased production of luminol enhanced chemiluminescence by the inflamed colonic mucosa in patients with ulcerative colitis. Gut 1993; 34: 1191-7.

3 Babbs CF. Oxygen radicals in ulcerative colitis. Free Rad Biol Med 1992; 13: 169-82.

4 Grisham MB. Oxidants and free radicals in inflammatory bowel disease. Lancet 1994; 344: 859-61.

5 Repine JE, Eaton JW, Anders MW, Hoidal JR, Fox RB. Generation of hydroxyl radical by enzymes, chemicals and human phagocytes in vitro. Detection with the antiinflammatory agent dimethyl sulphoxide. $\mathcal{F}$ Clin Invest 1979; 64: 1642-51.

6 Grisham MB, Granger DN. Neutrophil-mediated mucosal injury. Role of reactive oxygen metabolites. Dig Dis Sci 1988; 33 (suppl): 6-15S.

7 Hawkey CJ, Rampton DS. Prostaglandins and the gastrointestinal mucosa: are they important in its function, disease, or treatment? Gastroenterology 1985; 89: 1162-82.

8 Grisham MB, MacDermott RP, Deitch EA. Oxidant defense mechanisms in the human colon. Inflammation 1990; 14: 669-80.

9 Aruoma OI, Kaur H, Halliwell B. Oxygen free radicals and human diseases. $\mathcal{F}$ Roy Soc Health 1991; 111: 172-7.

10 Chakraborti S, Gurtner GH, Michael JR. Oxidant-mediated activation of phospholipase A2 in pulmonary endoactivation of phospholipase A2 in pul
thelium. Am f Physiol 1989; 257: L430-7. 
11 Reindeau D, Denis D, Choo LY, Nathaniel DJ. Stimulation of 5-lipoxygenase activity under conditions which promote lipid peroxidation. Biochem $f$ 1989; 263 565-72.

12 Zimmerman BJ, Grisham MB, Granger DN. Role of oxidants in ischaemia/reperfusion-induced granulocyte infiltration. Am ₹ Physiol 1990; 258: G185-G90.

13 Schreck R, Rieber P, Baeuerle PA. Reactive oxygen intermediates as apparently widely used messengers in the activation of NFkappaB transcription factor and HIV-1. EMBO ₹ 1991; 10: 2247-58.

14 Amstad PA, Krupitza G, Cerutti PA. Mechanism of c-fos induction by active oxygen. Cancer Res 1992; 52: 3952-60.

15 Ahnfelt-Ronne I, Nielsen OH, Christensen A, Langholz E, Binder V, Riis P. Clinical evidence supporting the radical scavenger mechanism of 5-aminosalicylic acid. Gastroenterology 1990; 98: 1162-9.

16 Baltch AL, Hammer MC, Smith RP, et al. Comparison of the effect of three adrenal corticosteroids on human granulocyte function against Pseudomonas aeruginosa. Trauma 1986; 26: 523-33.

17 Emerit J, Pelletier S, Likforman J, Pasquier C, Thuillier A. Phase II trial of superoxide dismutase (CuZn SOD) in the treatment of Crohn's disease. Free Radic Res Commun 1991; 12-13 (Pt 2): 563-9.

18 Levin KE, Pemberton JH, Phillips SF, Zinsmeister AR, Pezim ME. Role of oxygen free radicals in the etiology of pouchitis. Dis Colon Rectum 1992; 35: 452-6.

19 Keshavarzian A, Sedghi S, Kanofsky J, et al. Excessive production of reactive oxygen metabolites by inflamed colon: analysis by chemiluminescence probe. Gastroenterology 1992; 103: 177-85.

20 Yoshikawa T, Takahashi S, Kondo M. Possible role of free radicals in chronic inflammation in the gut. EXS 1992 62: 353-8.

21 Sharon P, Stenson WF. Metabolism of arachonic acid in acetic acid. Similarity to human inflammatory bowel disease. Gastroenterology 1985; 88: 55-63.

22 Keshavarzian A, Morgan G, Sedghi S, Gordon JH, Doria $M$. Role of reactive oxygen metabolites in experimental colitis. Gut 1990; 31: 786-90.

23 Keshavarzian A, Haydek J, Zabihi R, Doria M, D'Astice M, Sorensen JRJ. Agents capable of eliminating reactive oxygen species: catalase, WR-2721, Cu(II)2(3,5-DIPS) decrease experimental colitis. Dig Dis Sci 1992; 37: 1866-73.

24 Simmonds NJ. Reactive oxygen metabolites and inflammatory bowel disease [Doctor of Medicine, Thesis]. Oxford: 1992.

25 Simmonds NJ, Blake DR, Rampton DS. The effects of aminosalicylates and potential new drugs on mucosal reactive oxygen metabolite production in ulcerative colitis. Gut 1992; 33 (suppl): S7.

26 MacPherson BR, Pfeiffer CJ. Experimental production of diffuse colitis in rats. Digestion 1978; 17: 135-50.

27 Morris GP, Beck PL, Herridge MS, Depew WT, Szewczuk MR, Wallace JL. Hapten-induced model of chronic inflammation and ulceration in the rat colon Gastroenterology 1989; 96: 795-803.

28 Allen RC. Phagocyte oxygenation activities: quantitative analysis based on chemiluminescence. In: Scholmerich J, Andreeson R, Knapp A, Ernst M, Woods WG, eds Bioluminescence and chemiluminescence. New York: Wiley, 1986: 13-22.

29 Halliwell B, Gutteridge JMC. Free radicals in biology and medicine. (2nd ed.) Oxford: Clarendon, 1989.

30 Heikkila $R$. Inactivation of superoxide dismutase by diethylcarbamate. In: Greenwald $\mathrm{R}$, ed. CRC handbook of methods for oxygen radical research. Boca Raton, Florida: methods for oxygen radical rese

31 Snedecor GW. Statistical methods applied to agriculture and biology. 5th ed. Iowa, USA: The Iowa State University Press, 1956: 62

32 Staerk-Laursen L, Stokholm M, Bukhave K, Rask-Madsen J, Lauritsen $\mathbf{K}$. Disposition of 5 -aminosalicylic acid by olsalazine and three mesalazine preparations in patient with ulcerative colitis: comparison of intraluminal colonic concentrations, serum values, and urinary excretion. Gut 1990; 31: 1271-6.

33 Maridonneau-Parini I, Errasfa $M$, Russo-Marie F. Inhibition of O2-generation by dexamethasone is mimicked by lipocortin I in alveolar macrophages. $\mathcal{f}$ Clin Invest 1989; 83: 1936-40.

34 Nurcombe HL, Edwards SW. Role of myeloperoxidase in intracellular and extracellular chemiluminescence of neutrophils. Ann Rheum Dis 1989; 48: 56-62.

35 Hallett MB, Campbell AK. Two distinct mechanisms for stimulation of oxygen-radical production by polymorphonuclear phagocytes. Biochem $\mathcal{f}$ 1983; 216 459-65.

$36 \mathrm{McCord}$ JM. Oxygen-derived free radicals in postischaemic tissue injury. N Engl f Med 1985; 312: 159-63.

37 Wakefield AJ, Sawyerr AM, Dhillon AP, et al. Pathogenesis of Crohn's disease: multifocal gastrointestinal infarction. Lancet 1989; ii: 1057-62.
38 Oshitani N, Kitano A, Okabe H, Nakamura S, Matsumoto $\mathrm{T}$, Kobayashi $\mathrm{K}$. Location of superoxide anion generation in human colonic mucosa obtained by biopsy. Gut 1993; 34: 936-8.

39 Mulder TP, Verspaget HW, Janssens AR, deBruin PA, Pena AS, Lamers CB. Decrease in two intestinal copper/zinc containing proteins with antioxidant function in inflammatory bowel disease. Gut 1991; 32: 1146-50.

40 Niwa Y, Somiay K, Michelson AM, Puget K. Effect of liposomal-encapsulated superoxide dismutase on active oxygen-related disorders. Free Radic Res Commun 1985; 1: 137-53.

41 Gorecki M, Beck Y, Hartman JR, et al. Recombinant human superoxide dismutates: production and potential therapeutical uses. Free Radic Res Commun 1991; 12-13 Pt 1: 401-10.

42 Parizada B, Weber MM, Nimrod A. Protective effects of human recombinant MnSOD in adjuvant arthritis and bleomycin-induced lung fibrosis. Free Radic Res Commun 1991; 15: 297-301.

43 Susuki M, Grisham MB, Granger DN. Leukocyteendothelial adhesive interactions: role of xanthine oxidase-derived oxidants. I Leukoc Biol 1991; 50: 488-94.

44 Bilota JJ, Wayne JD. Hydrogen peroxide enteritis: the "snow white" sign. Gastrointest Endosc 1989; 35: 428-30.

45 Aruoma OI, Halliwell B, Hoey BM, Butler J. The antioxidant action of $\mathrm{N}$-acetylcysteine: its reaction with antioxidant action of $\mathrm{N}$-acetylcysteine: its reaction with hydrogen peroxide, hydroxyl radical, superoxide and
hypochlorous acid. Free Rad Biol Med 1989; 6: 593-7.

46 Nishikimi $M$. Oxidation of ascorbic acid with superoxide anion generated by the xanthine-xanthine oxidase system. Biochem Biophys Res Commun 1975; 63: 463-8.

47 Halliwell B, Wasil M, Grootveld M. Biologically significan scavenging of the myeloperoxidase-derived oxidan hypochlorous acid by ascorbic acid. FEBS Letts 1987 ; 213: 15-8.

48 Pettit SH, Shaffer JL, Johns CW, Bennett RJM, Irving MH. Ascorbic acid absorption in Crohn's disease. Studies using L-[carbonyl-14C] ascorbic acid. Dig Dis Sci 1989; 34: 559-60.

49 Fernandez-Banares $F$, Mingorance $M D$, Esteve $M$ Essential antioxidants in active idiopathic colitis. In Goebell H, Ewe K, Malchov H, Koebel C, eds. Inflammatory bowel disease. Progress in basic research and clinical implications. Lancaster: Kluwer Academic, 1991: 404.

50 Rowley DA, Halliwell B. Formation of hydroxyl radicals from hydrogen peroxide and iron salts by superoxide- and ascorbate dependent mechanisms: relevance to the pathology of rheumatoid disease. Clin Sci 1983; 64: 649-53.

51 Clemens JA, Saunders RD, Ho PP, Phebus LA, Panetta JA. The antioxidant LY231617 reduces global ischaemic neuronal injury in rats. Stroke 1993; 24: 722-3.

52 Phillis JW, Sen S, Cao X. Amflutizole, a xanthine oxidase inhibitor, inhibits free radical generation in the ischemic/ inhibitor, inhibits free radical generation in the ischemic/ reperfused

53 Dhillon AP, Anthony A, Sim R, et al. Mucosal capillary thrombi in rectal biopsies. Histopathology 1992; 21: 172-33

54 Sharanov BP, Govorova NJ, Lyzlova SN. Serum protein degradation by hypochlorite. Biochem Int 1989; 19: $27-35$.

55 Klebanoff S. Oxygen metabolites from phagocytes. In: Gallin J, Goldstein I, Snyderman R, eds. New York: Raven Press, 1992: 541-88.

56 Dallegri F, Ottonello L, Ballestrero F, Ferrando F, Patrone F. Cytoprotection against neutrophil derived hypochlorous acid: a potential mechanism for the hypochlorous acid: a potential mechanism for the colitis. Gut 1990; 31: 184-6.

57 Green TR, Fellman JH, Eicher AL, Pratt KL. Antioxidant role and subcellular location of hypotaurine and taurine in human neutrophils. Biochim Biophys Acta 1991; 1073: 91-7.

58 Beilke MA, Collins-Lech C, Sohnle PG. Effects of dimethyl sulphoxide on the oxidative function of human neutrophils. $f$ Lab Clin Med 1987; 110: 91-6.

59 Miyachi Y, Yashioka A, Imamura S, Niwa Y. Effect of sulphasalazine and its metabolites on the generation of reactive oxygen species. Gut 1987, 28: 190-5.

60 Craven PA, Pfanstiel J, Saito R, DeRubertis FR. Actions of sulphasalazine and 5-aminosalicylic acid as reactive oxygen scavengers in the suppression of bile acid induced increases in colonic epithelial loss and proliferative increases in colonic epithelial loss and proli

61 Aruoma OI, Wasil M, Halliwell B, Hoey BM, Butler J. The scavenging of oxidants by sulphasalazine and its metabolites. A possible contribution to their antiinflammatory effects? Biochem Pharmacol 1987; 36: 3739-42.

62 Grisham MB. Effect of 5-aminosalicylic acid in ferrous sulphate-mediated damage to deoxyribose. Biochem Pharmacol 1990; 39: 2060-3. 